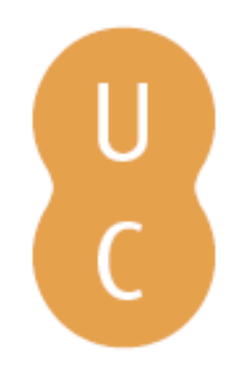

\title{
pommalina
}

\section{Vestígios do tsunami de 1755: um indicador negligenciado no litoral de Portugal continental?}

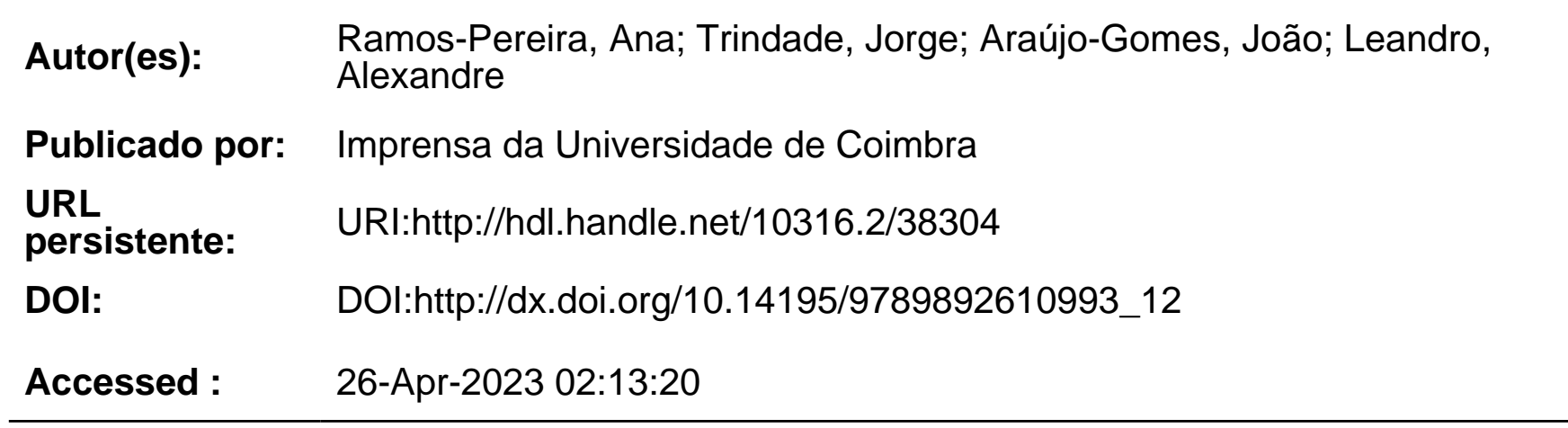

A navegação consulta e descarregamento dos títulos inseridos nas Bibliotecas Digitais UC Digitalis, UC Pombalina e UC Impactum, pressupõem a aceitação plena e sem reservas dos Termos e Condições de Uso destas Bibliotecas Digitais, disponíveis em https://digitalis.uc.pt/pt-pt/termos.

Conforme exposto nos referidos Termos e Condições de Uso, o descarregamento de títulos de acesso restrito requer uma licença válida de autorização devendo o utilizador aceder ao(s) documento(s) a partir de um endereço de IP da instituição detentora da supramencionada licença.

Ao utilizador é apenas permitido o descarregamento para uso pessoal, pelo que o emprego do(s) título(s) descarregado(s) para outro fim, designadamente comercial, carece de autorização do respetivo autor ou editor da obra.

Na medida em que todas as obras da UC Digitalis se encontram protegidas pelo Código do Direito de Autor e Direitos Conexos e demais legislação aplicável, toda a cópia, parcial ou total, deste documento, nos casos em que é legalmente admitida, deverá conter ou fazer-se acompanhar por este aviso.

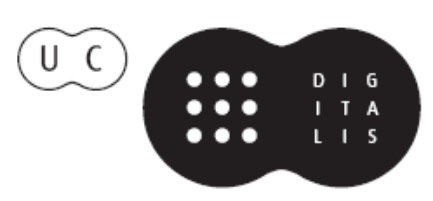




\section{TERRAMOTO DE \\ LISBOA DE 1755}

\section{O QUE APRENDEMOS \\ 260 ANOS DEPOIS?}

LUCIANO LOURENÇO

ÂNGELA SANTOS

(COORDS.)

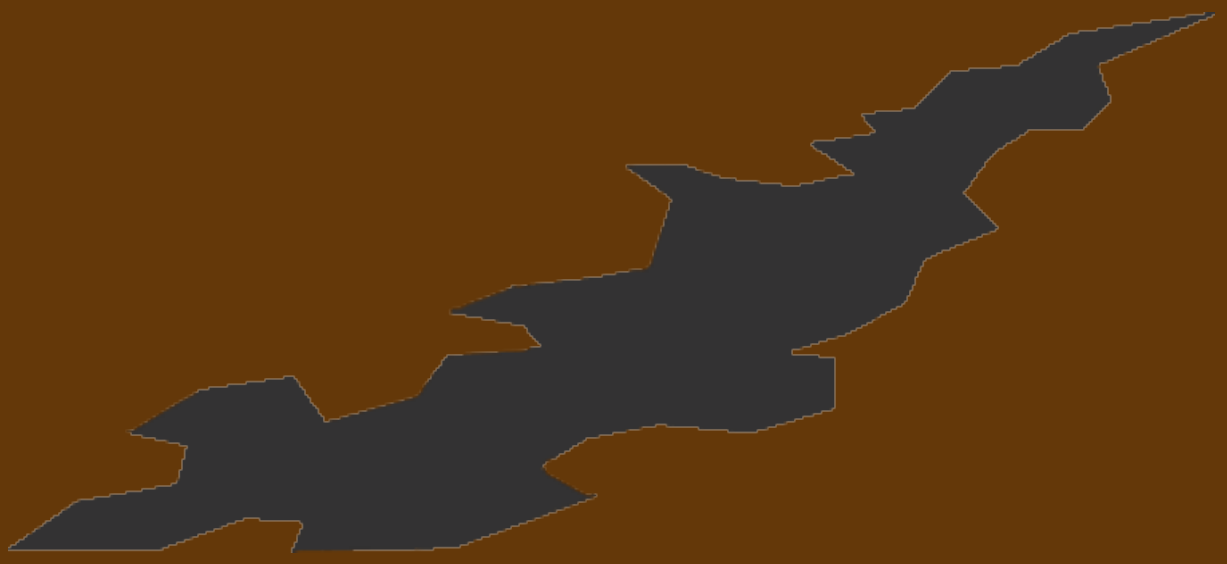

IMPRENSA DA UNIVERSIDADE

DE COIMBRA

COIMBRA

UNIVERSITY

PRESS 


\title{
VESTÍGIOS DO TSUNAMI DE 1755: UM INDICADOR NEGLIGENCIADO NO LITORAL DE PORTUGAL CONTINENTAL? \\ 1755 TSUNAMI REMNANTS: A NEGLECTED INDICATOR OF COASTAL HAZARD IN PORTUGAL MAINLAND?
}

\begin{abstract}
Ana Ramos-Pereira Centro de Estudos Geográficos, IGOT da Universidade de Lisboa anarp@campus.ul.pt

Jorge Trindade Centro de Estudos Geográficos, IGOT da Univ. de Lisboa / Univ. Aberta jorgetrd@uab.pt

João Araújo-Gomes Centro de Estudos Geográficos, IGOT da Universidade de Lisboa joaopgomes@campus.ul.pt

Alexandre Leandro Centro de Estudos Geográficos, IGOT da Universidade de Lisboa alexandreleandro@campus.ul.pt
\end{abstract}

Sumário: Os tsunamis podem originar fenómenos de inundações marinhas extremas em áreas costeiras com efeitos devastadores. Este capítulo refere parte da investigação que tem vindo a ser desenvolvida sobre os vestígios do tsunami de 1755 em Portugal continental, talvez o mais devastador registado até hoje no nosso território. O contexto geoestrutural do país torna-o susceptível a este fenómeno extremo que deixou marcas na costa $\mathrm{W}$ e $\mathrm{S}$ de Portugal continental. A multiplicidade tipológica dos registos de tsunami é comprovada por diversos trabalhos, entre os quais dois estudos distintos, um em Lisboa (novo Museu dos Coches) e outro em Odemira (na área de Malhão).

Palavras-chave: Tsunami, 1755, Belém, Malhão, tsunamito 


\begin{abstract}
Tsunami may cause extreme flooding of coastal areas with devastating effects. This chapter covers part of the research carried out on the remnants of the 1755 tsunami in Portugal mainland, perhaps the most devastating in our territory. The country geostrutural context makes it prone to this extreme event, which left its remains on the Western and Southern coast of Portugal mainland, shown by several research studies. The typological multiplicity of tsunami records is proven in this study by two distinct works, one in Lisbon (new Coach Museum) and the other in Odemira (in Malhão area).
\end{abstract}

Keywords: Tsunami, 1755, Belém, Malhão, tsunamiite

\title{
Introduçáo
}

Tsunamis constituem eventos hidrodinâmicos extremos, gerados por diversos mecanismos físicos, sejam sismos, atividade vulcânica, grandes deslizamentos ou queda de meteoritos, que ocorrem em espaço marinho e cujas ondas se propagam a várias centenas de quilómetros por hora, podendo atingir as terras emersas. Aí, em águas pouco profundas, a velocidade da(s) onda(s) de tsunami (maremoto, como eram chamados pelos nossos descobridores no século XVI) diminui, mas a altura da onda(s) aumenta, podendo atingir dezenas de metros. O seu efeito destruidor no território litoral deve-se sobretudo à energia que essas ondas possuem, mesmo que a sua altura seja da ordem dos poucos metros.

Aqui serão referidos apenas os vestígios reconhecidos do tsunami de 1755 , ocorrido na sequência do sismo de magnitude 8,5 (8,5-9 de acordo com M.A. Gutscher et al., 2006). A altura estimada da onda de tsunami que se lhe seguiu terá sido de 10-12 m no Cabo de S. Vicente, 4,28 m em Oeiras e $6 \mathrm{~m}$ em Lisboa (M. A. Baptista et al. 1996,1998, 2003). O seu efeito destruidor em Lisboa, e também no Algarve, foi objeto de inúmeros relatos de viajantes, de pinturas, de descriçôes em documentos oficiais e arquivos paroquiais, sem contudo ser conhecida, até ao final da década passada, a sua assinatura sedi- 
mentar no espaço urbano de Lisboa (A. Ramos-Pereira et al., 2013). A maior parte das investigaçóes realizadas em Portugal evidenciou claramente os vestígios de tsunami no Algarve.

\section{Posição geoestrutural de Portugal continental}

A posição geo-estrutural de Portugal continental torna-o suscetível a sismos com epicentros submarinos, alguns de elevada magnitude, geradores de tsunamis.

Situado na proximidade da complexa fronteira tectónica entre a placa africana e a microplaca ibérica (falha Açores-Gibraltar), ou da falha Marquês de Pombal, ao largo da costa alentejana ou ainda do início de uma subdução incipiente na margem ibérica ocidental (A. Ribeiro et al., 1996; J. F. Borges et al., 2001; P. Terrinha et al., 2003), o território litoral de Portugal continental ao sul de Peniche é um território perigoso, suscetível a este fenómeno. Esta parte do território português continental contém extensas áreas urbanizadas, como a área Metropolitana de Lisboa e o Algarve, onde este risco não deve ser negligenciado.

\section{Os vestígios de tsunami na costa de Portugal continental}

\section{A sua posição geomorfológica}

Os vestígios de tsunami até agora reconhecidos ocupam quatro contextos geomorfológicas distintos associados:

(i) ao enchimento sedimentar dos estuários

(ii) a sistemas de barreira

(iii) a lagunas (lagoas costeiras)

(iv) a topos de arribas 
No primeiro caso os sedimentos resultam do galgamento da(s) ondas de tsunami (i). Como o tsunami ocorreu há 260 anos, os sedimentos que o testemunham estáo soterrados por outros mais recentes ou mesmo por aterros e podem distar dezenas de metros para o interior da atual linha de costa. Por esse motivo, a sua presença só pode ser revelada após a realização de sondagens ou aberturas de sanjas. Esses testemunhos podem encontrar-se a diferentes profundidades no interior de vários estuários, dependendo da taxa de sedimentação subsequente, que é variável consoante as características do escoamento fluvial e a disponibilidade sedimentar. Este tipo de vestígios foi revelado em áreas de estuários não artificializados, a maior parte dos quais em áreas rurais ou em áreas litorais protegidas. Citem-se, a título de exemplo, os pequenos estuários assoreados da Boca do Rio (R. A. Hindson e C. Andrade 1999; S. Dawson et al. 1995) e Martinhal (Andrade et al., 1997; S. Kortekaas e A. Dawson, 2007; P. Costa et al., 2011). Só excecionalmente foram encontrados em área urbana (A. Ramos-Pereira, 2013; fig. 1).

A morfologia específica deixada pelos galgamentos tsunâmicos (ii) foi também referenciada no sistema de barreira da Formosa, no Algarve (C. Andrade, 1990 e 1992), evidenciada pela rotura da barreira arenosa, bem como pela injecção de sedimentos arenosos associados a galgamentos que criaram deltas coalescentes.

Mais recentemente começaram a surgir testemunhos no enchimento de lagunas (iii), como nos Salgados, no Algarve, ou na paleolaguna de Santa Rita, na Estremadura, a NW de Lisboa (P. Costa et al., 2014, A. Ramos Pereira, em publicação).

Sáo escassos os vestígios encontrados no topo das arribas (iv). Estes estão expostos e podem ser confundidos com sedimentos de temporal (citem-se a título de exemplo Costa et al, 2008; Goto et al., 2009; Shah-Houssaini et al., 2013). Grandes blocos provavelmente colocados naquela posição por onda(s) de tsunami foram registados na plataforma de Cascais, a sul do Cabo da Roca, e em Malhão, no Alentejo (A. Scheffers e D. Kelletat, 2005 e A. Ramos Pereira et al., 2009, respetivamente).

Não deve deixar de salientar-se que, apesar de apenas se tratar aqui da área emersa, são conhecidos vestígios ao largo, na margem portuguesa, traduzidos 
em deslizamentos desencadeados pela actividade tectónica nas falhas Marquês de Pombal e Pereira de Sousa (P. Terrinha et al., 2003; Abrantes et al., 2005).

\section{A assinatura sedimentar}

Do exposto se pode deduzir que a assinatura sedimentar dos tsunamis é distinta consoante o seu contexto geomorfológico, em estuários e costas arenosas e em costas rochosas, com arriba e rampas.

Os sedimentos em estuários e costas arenosas distinguem-se dos enquadrantes por possuírem as seguintes caraterísticas:

(i) Composição textural específica, nomeadamente mistura de sedimentos de diversas proveniências e com várias composiçôes, a matriz é em geral arenosa, mais grosseira (C. Trog et al.,2015) e pode ter uma distribuição bimodal (A. Scheffers e D. Kelletat, 2004) é também possível encontrar bolas de argila envolvidas por areia, como sucede no Martinhal (S. Kortekaas e A. G. Dawson, 2007); os grãos de quartzo podem também exibir marcas específicas, embora até agora os resultados da investigação não tenham sido concludentes (P. Costa et al., 2012). Os cortejos de minerais pesados indicam também particularidades específicas (P. Costa et al., 2015).

(ii) Estrutura marcada por disposição caótica dos vários componentes no seio do sedimento depositado pelo tsunami (Hindson et al., 1996), como sucede em Belém, Lisboa - tsunamito (A. Ramos-Pereira etal., 2013).

(iii) Composição geoquímica marcada pela presença de elementos que revelam a presença de água salgada, como por exemplo na laguna dos Salgados, no Algarve (Moreira et al., 2014; P. Costa et al., 2014; fig 1).

(iv) Presença de macrorrestos, nomeadamente fragmentos de conchas (E. Bryant et al., 2008).

(v) Presença de microfauna como diatomáceas, foraminíferos, ostracodos específicos descritos na bibliografia internacional e também estudados, 
por exemplo, no Martinhal (S. Kortekaas e A. G. Dawson, 2007) e nos estuários de Alvor e Alcantarilha (Trog et al, 2013).

(vi) Presença de um cortejo polínico revelador da mistura de materiais movimentados pelo tsunami (A. G. Dawson, 1995).

Uma única destas caraterísticas é insuficiente para atribuir a génese tsunâmica aos sedimentos. É sobretudo o resultado do cruzamento de 2 ou mais destas caraterísticas que revela a sua originalidade em relação aos sedimentos enquadrantes (Vott et al., 2011).

Não deve ainda deixar de se referir que, no interior dos estuários, além dos sedimentos relativamente finos que constituem no geral os tsunamitos, também se encontram blocos de grandes dimensôes com marcas de biocorrosão por seres vivos marinhos, a centenas de metros para o interior da foz, como sucede no estuário do Barranco de Benaçoitão e das Furnas, no Algarve (Oliveira et al., 2008; P. Costa et al., 2008; fig.1).

Os sedimentos de tsunamito atribuídos ao fenómeno ocorrido em 1755 têm sido datados por radiocarbono e luminescência opticamente estimulada (P. P. e Cunha et al. 2010).

Os sedimentos testemunhos de tsunami no topo das arribas são exclusivamente (até ao momento presente das investigaçóes) acumulaçóes de grandes blocos. Pela sua posição alcandorada no topo da arriba, os testemunhos sedimentares são presa da erosão hídrica e eólica, pelo que apenas restam os grandes blocos, esparsos e isolados no topo da arriba ou formando amontoados ao longo da linha de costa. Estes blocos provêm das áreas submersas próximas ou foram arrancados pelo tsunami à face da arriba. Estes últimos, porque estiveram expostos, contêm marcas da meteorização a que foram sujeitos e alguns deles estão no topo da arriba em posição invertida.

\section{O tsunamito de Belém (Lisboa)}

Há cerca de 260 anos, as áreas marginais ao Rio Tejo, na área de Belém, exibiam uma paisagem completamente diversa da atual. Tratava-se de uma 


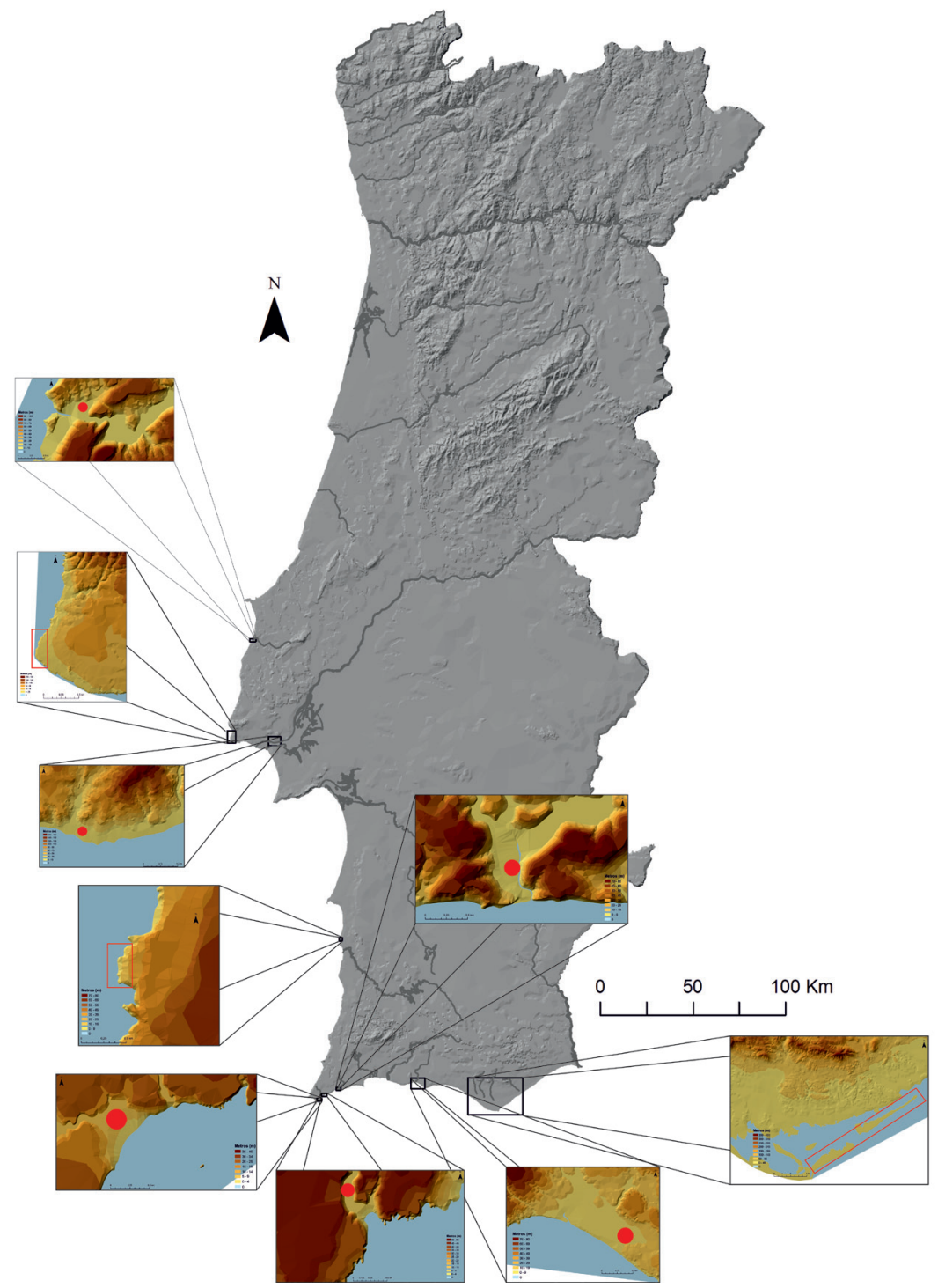

Fig. 1 - Locais citados no texto, onde foram registados vestígios do tsunami de 1755. $\mathrm{Na}$ costa ocidental de norte para sul: estuário do Rio Alcabrichel; Plataforma de Cascais; Belém; Malhão. Na costa meridional de oeste para este: Martinhal; Barranco de Benaçoitão e Furnas; Salgados; Sistema de Barreira da Formosa.

Fig. 1 - Sites mentioned in the text where the 1755 tsunami traces were recorded. In the Western coast, from North to South: Rio Alcabrichel estuary; Cascais platform; Belém; Malhão. In the Southern coast, from West to East: Martinhal; Barranco de Benaçoitão e Furnas; Salgados; Barrier Islands of Formosa. 
fímbria de planície, marginal ao rio, construída por aluvióes das várias ribeiras afluentes do Tejo e do próprio Rio Tejo e por uma praia estuarina e áreas de sapal (fig. 2). A Ribeira da Ajuda, por exemplo, cujo escoamento se fazia aproximadamente onde existe hoje a calçada com o mesmo nome, desaguava aí. A área era marcadamente rural e com baixa densidade populacional (A. Ramos-Pereira et al., 2014).

As obras para a construçấo do novo Museu dos Coches, que se iniciaram em 2008, constituíram uma oportunidade única para a reconstituição dessa paleopaisagem e das vicissitudes a que foi sujeita.

A sequência de sedimentos postos a descoberto pela abertura de várias sanjas mostrou, da base para o topo (fot. 1):

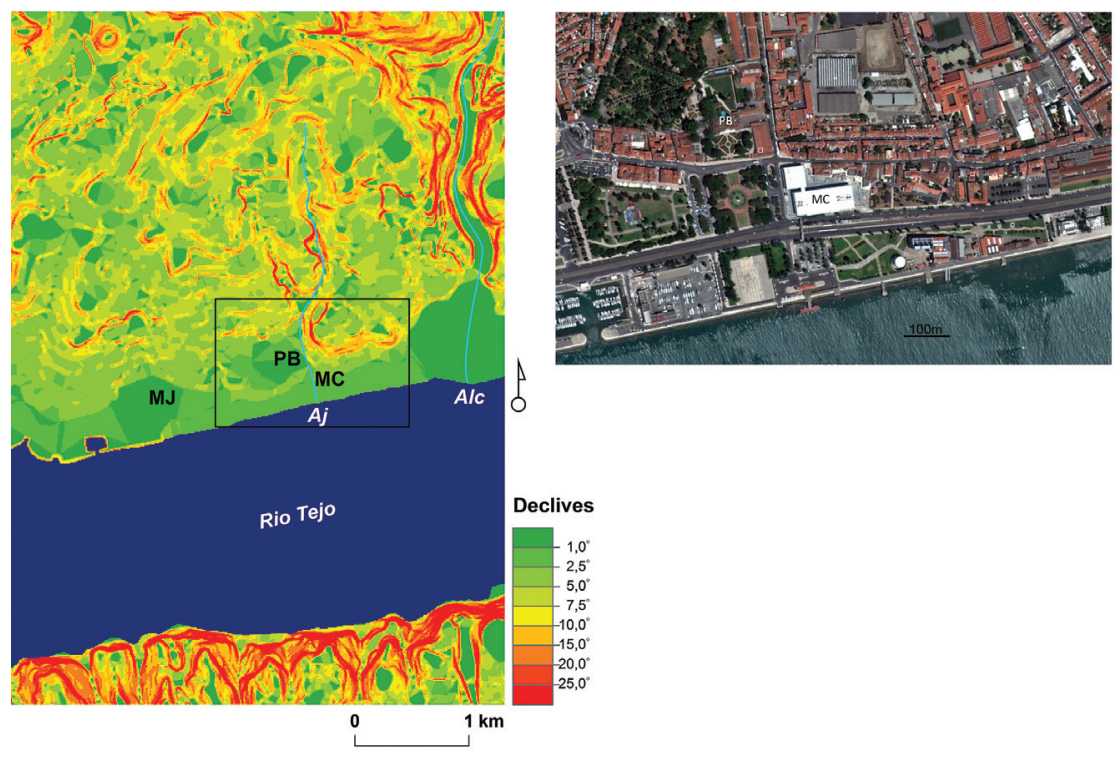

Fig. 2 - O estuário do Tejo na área de Belém. A) MC - novo Museu dos Coches; PB - Palácio de Belém; Aj - Ribeira da Ajuda; Alc - Ribeira de Alcântara. MJ - Mosteiro dos Jerónimos. B) Imagem de 25 de maio de 2013, extraída do Google Earth, mostra o Museu dos Coches.

Fig. 2 - The estuary of the Tagus in the Belém area A) MC - New Coach Museum; PB - Palace of Belém; Aj-Ajuda stream; Alc-Alcantara stream; MJ - Monastery of Jerónimos. B) Image taken May 25, 2013, taken from Google Earth, shows the new

Coach Museum. 
(i) Sedimentos cascalhentos rolados, inclinados para o Tejo e com uma matriz arenosa quase inexistente; estes sedimentos inteiramente constituídos por basalto provêm da vertente do estuário (antiga arriba) e revelam ter sido rolados in situ, pela ondulação que afeta (ainda hoje) aquela parte terminal do estuário; trata-se de uma praia estuarina cascalhenta;

(ii) Areia amarelada, média a fina, com finos leitos escuros, que termina em bisel para terra; a estrutura destas areias mostra que está também ligeiramente inclinada para o Tejo; este leito evidencia uma praia estuarina arenosa;

(iii) Material cascalhento, diferente do da base por os seixos e blocos não serem apenas de basalto, mas também de calcário, dispostos de forma caótica, envolvidos numa matriz de areia grosseira a muito grosseira, relativamente abundante, com fragmentos de conchas de bivalves visíveis a olho nu e manchas escuras, por vezes com formas caprichosas; este depósito é interpretado como um tsunamito (A. Ramos-Pereira et. al, 2013);

(iv) Areia idêntica a (ii), de uma praia estuarina;

(v) Aterro que sela a sequência sedimentar.

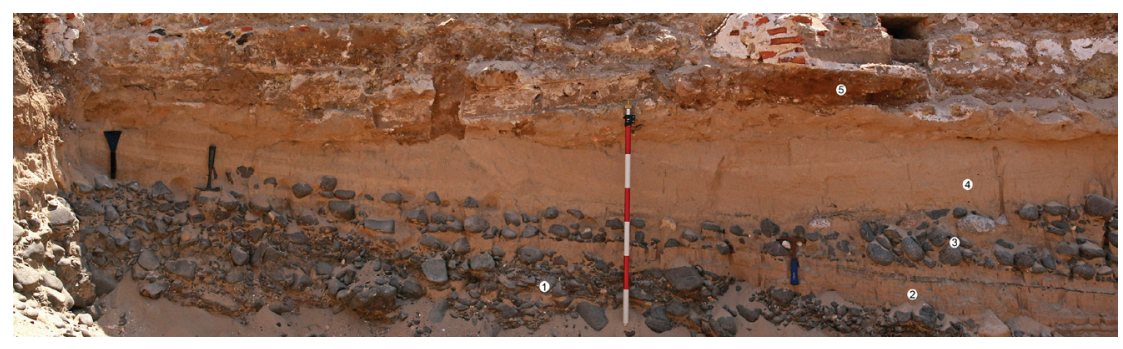

Fot. 1 - Sequência sedimentar posta a descoberto numa das sanjas aberta em 2008, na fase exploratória de avaliaçáo do tipo de substrato. Da base para o topo, 1 - praia cascalhenta, 2 - praia estuarina, 3 - tsunamito, 4 - praia estuarina, 5 - aterro, de acordo com a referência no texto.

Photo. 1 - Sedimentary sequence lay bare in a trench opened in 2008, for exploratory evaluation of the substrate type. From the bottom to the top, 1 - gravel beach 2 - estuarine beach, 3 -tsunamite, 4 - estuarine beach, 5 -landfill, according to the reference in the text. 
Importa salientar a presença de areias negras em (ii) e (iii), dispostas em finos leitos ou que constituem manchas difusas. Trata-se de areias com elevado teor em minerais pesados, frequentes em algumas praias. A sua disposiçáo é, se não afetadas posteriormente por movimentos bruscos, idêntica às restantes areias (de quartzo). Porém, quando afetadas por estes movimentos, as areias de quartzo e de minerais pesados podem liquefazer-se, e a expulsão de bolhas de ar e de água (escape de fluidos) pode perturbar a sua disposição original. Não existem evidências regionais de outros fenómenos causadores de vibraçóes com magnitude suficiente para justificar as deformaçóes descritas, para além das vibrações sísmicas.

O terramoto de 1755, atingiu uma magnitude capaz de gerar um fenómeno hidrodinâmico altamente energético, competente para depositar o material referido em (iii). As deformaçóes encontradas nos leitos de areia escura são típicas de sedimentos afetados por fortes vibraçóes, que originam o escape de fluidos presentes nos depósitos. As réplicas que se seguiram ao grande terramoto e que atingiram magnitude estimada de 5,5, afetaram os depósitos (ii) e (iii tsunamito), que nessa altura já existiriam (fot. 2). A tipologia das deformaçóes encontradas, especialmente visíveis nos leitos de areia negra, está conforme com as geradas por sismos de grande magnitude (ver síntese sobre essas deformaçôes em J. Araújo-Gomes, 2013).

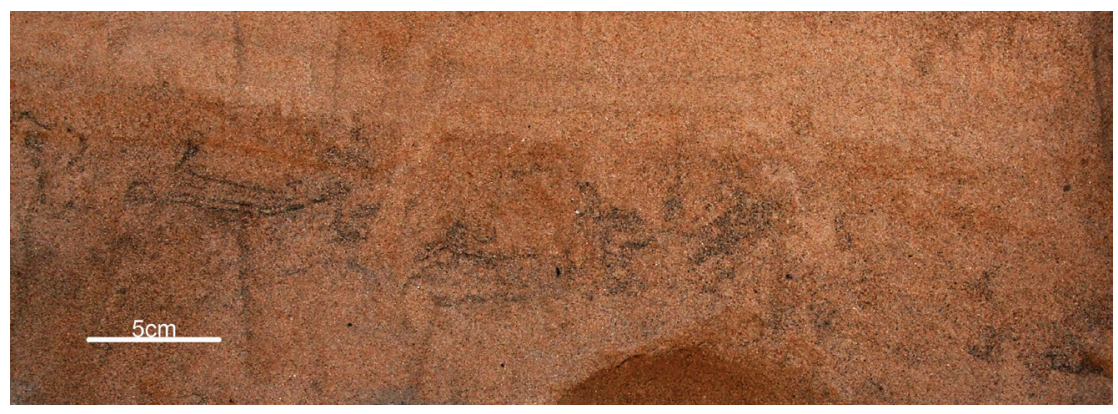

Fot. 2 - Pormenor das deformações dos leitos de minerais pesados por liquefação, na praia arenosa estuarina (ii).

Photo. 2 - Detail of heavy mineral layer deformations by liquefaction in estuarine sandy beach (ii). 
Num equivalente sedimentar lateral do tsunamito, foram encontrados ossos de dois esqueletos, cuja datação radiocarbono é consentânea com o século XVIII. No local estudado, o tsunami de 1755 apenas penetrou $400 \mathrm{~m}$ para norte em relação à posição atual do canal do Rio Tejo.

\section{O tsunamito de Malhão}

No litoral do Alentejo, ao norte de Vila Nova de Milfontes, no Parque Natural do Sudoeste Alentejano e Costa Vicentina, entre Angra da Barrela e Angra da Vaca (fig. 3), foram pela primeira vez registados por A. Ramos-Pereira (1987 e, posteriormente em 1990) a existência de grandes blocos no topo de uma arriba, cuja origem era desconhecida. Foi entáo colocada a hipótese de se tratar de blocos colocados por tsunami. Posteriormente, A. Ramos Pereira et al., 2009) retomaram este assunto, possuindo-se agora novos dados.

A área de Malháo é constituída por um extenso campo dunar, hoje carbonatado, que ocupa cerca de $20 \mathrm{~km}^{2}$ e penetra $3 \mathrm{~km}$ para o interior da atual linha de costa. A sua idade provável é do Plistocénico médio (OIS 6?; A. Ramos-Pereira e D. Angelucci, 2004), quando o nível do mar se situava abaixo do atual. Está cortado em arriba (foto) onde o eolianito (arenito dunar) chega a ter uma espessura superior a $15 \mathrm{~m}$. Está parcialmente coberto por areia solta. Localmente, o eolianito pode contatar com o mar por uma rampa (que atinge $13^{\circ} \mathrm{de}$ declive) ou estar cortado em arriba que pode ultrapassar $20 \mathrm{~m}$ (fot. 3).

Com o objetivo de avaliar o agente hidrodinâmico capaz de mover os grandes blocos e descartar a hipótese destes terem sido depositados por temporal, foi realizado um levantamento de campo com vista a caraterizar o modelado específico do promontório de Malhão (inteiramente constituído por eolianito). Foram também georeferenciados os blocos de grandes dimensões (43), avaliados os respetivos volumes e pesos, que atingem $9,5 \mathrm{~m}^{3}$ e 19 toneladas, respetivamente. Estes blocos são de eolianito e encontram-se desde o topo da arriba até $136 \mathrm{~m}$ para o interior da atual linha de costa e atingem $19 \mathrm{~m}$ de altitude (fot. 4). 
Merecem especial atenção alguns dos blocos por: (i) se localizarem a grande distância da linha de costa, até $134 \mathrm{~m}$ de distância para o interior; (ii) terem grande envergadura, até 19 toneladas, com um volume estimado de $9,5 \mathrm{~m}^{3}$, e se localizar a $116 \mathrm{~m}$ da linha de costa e a $15 \mathrm{~m}$ de altitude (fig. 7 e quadro 1 ) e (iii) por estarem em posiçấo invertida, demonstrando terem estado expostos a

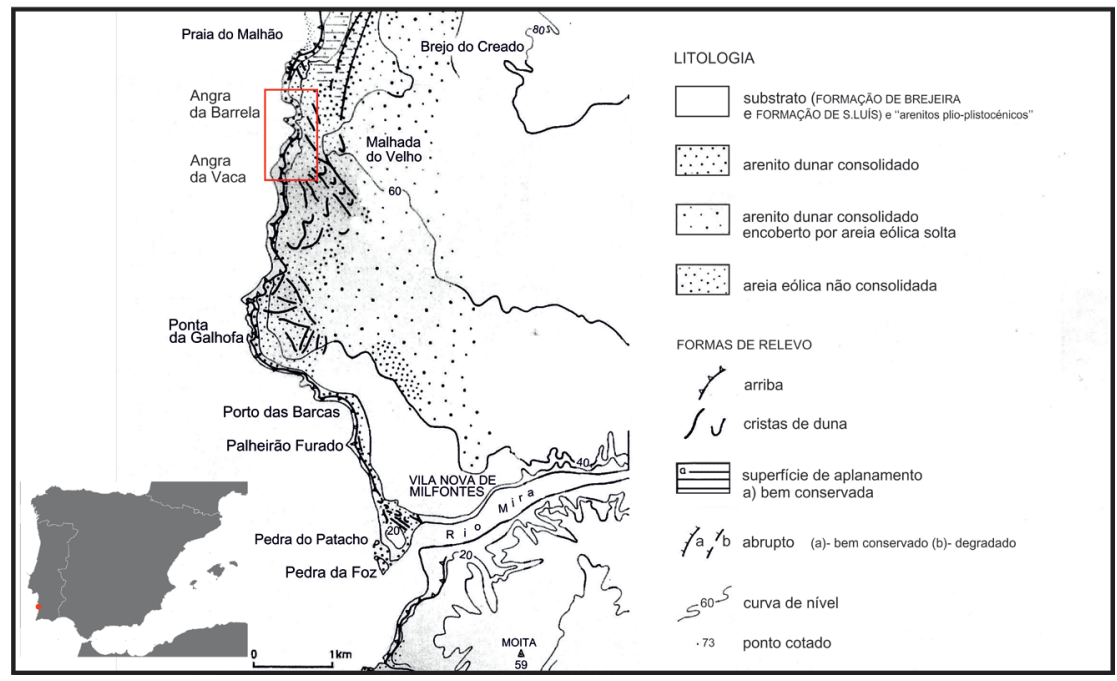

Fig. 3 - A área de Malháo. O retângulo indica o local onde foram encontrados os grandes blocos (Extraído de A. Ramos-Pereira 1987).

Fig. 3 - The Malhão area. The rectangle indicates the location where large blocks were found (From A. Ramos-Pereira 1987).
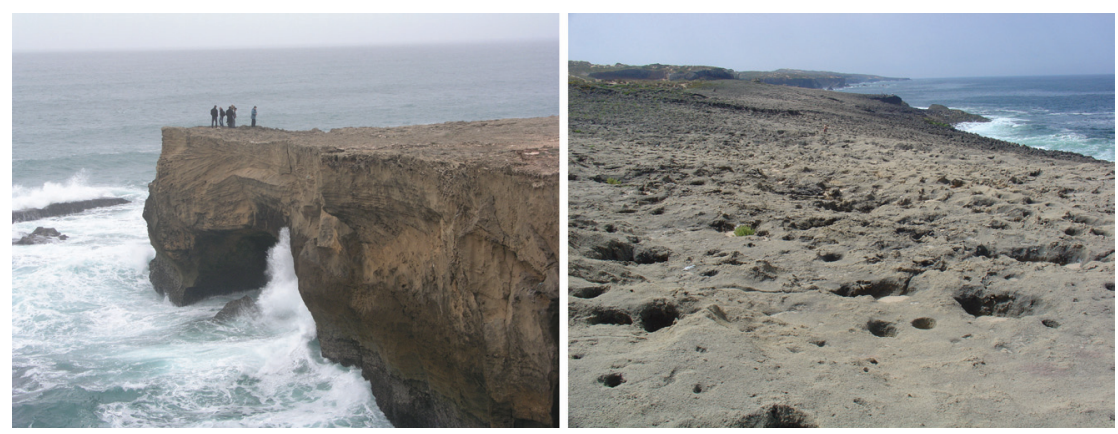

Fot. 3 - Os dois tipos de contacto do eolianito de Malhão com o mar (arriba e rampa).

Photo. 3 - The two types of coastline cut in the Malhão eolianite (cliff and ramp). 


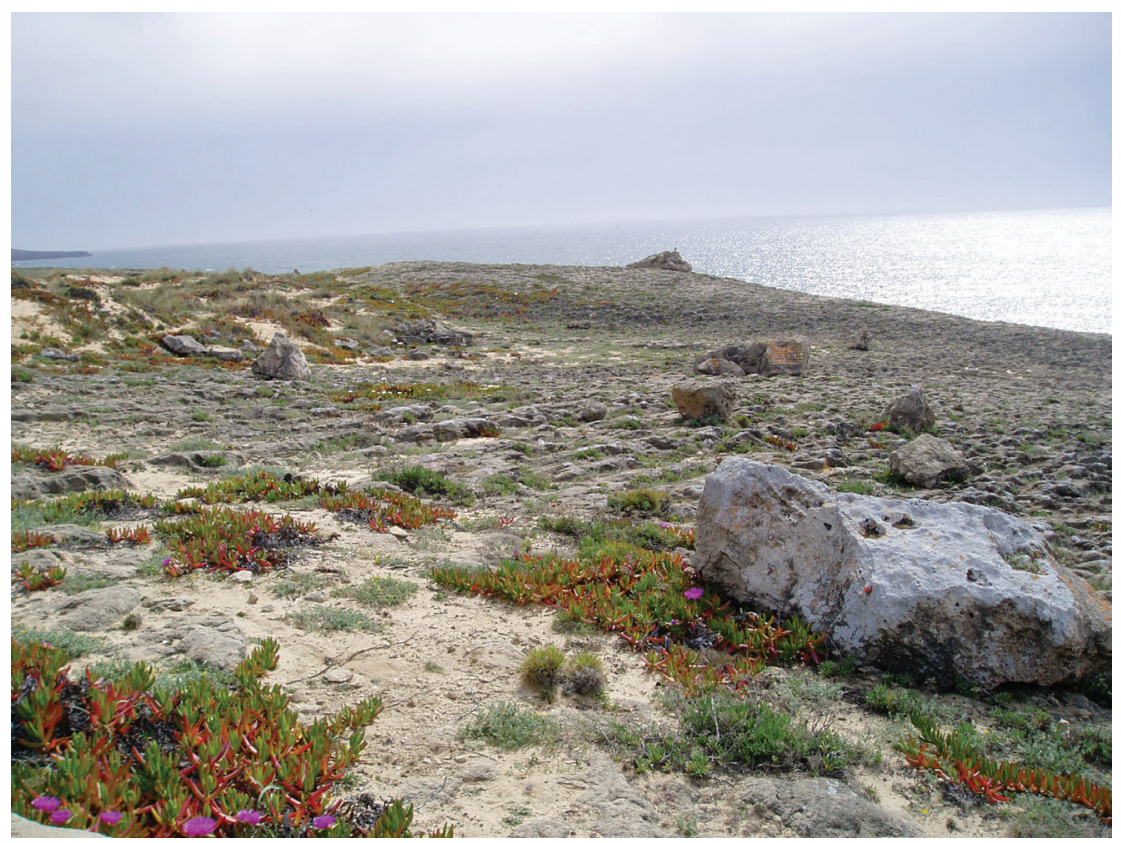

Fot. 4 - Aspeto da rampa talhada em eolianito com uma cobertura esparsa de grandes blocos, na área de Malhão.

Photo. 4 - Aspect of eolianite carved ramp with a sparse coverage of boulders in Malhão area.

meteorização subárea e sido destacados da arriba pela onda (fot. 5). O bloco invertido, localizado mais para o interior, situa-se a $106 \mathrm{~m}$ de distância da linha de costa.

Dos blocos que se destacam claramente pela elevada dimensão é possível determinar a altura de onda necessária para sua deslocação e deposição (J. Nott, 2003). Considerando a rede de fracturação, a natureza dos materiais e a morfologia da arriba, utilizou-se a formulação de J. Nott (2003) associada a blocos destacados do substrato para determinar as alturas de onda de tsunami $\left(H_{t}\right)$ e de onda de temporal $\left(H_{s}\right)$, cujos resultados se encontram expressos no QUADRo I.

$$
H_{t}=\frac{0,25\left(\rho_{s}-\rho_{w} / \rho_{w}\right) a}{C_{l}}
$$




$$
H_{s}=\frac{\left(\rho_{s}-\rho_{w} / \rho_{w}\right) a}{C_{l}}
$$

em que $\rho_{s}$ corresponde à densidade do bloco $\left(\mathrm{g} / \mathrm{cm}^{3}\right), \rho_{w}$ é o equivalente da densidade da água salgada para a costa portuguesa $(\mathrm{g} / \mathrm{ml})$, a corresponde ao eixo A do bloco (m) e $C l$ representa o coeficiente adimensional de levantamento do bloco.

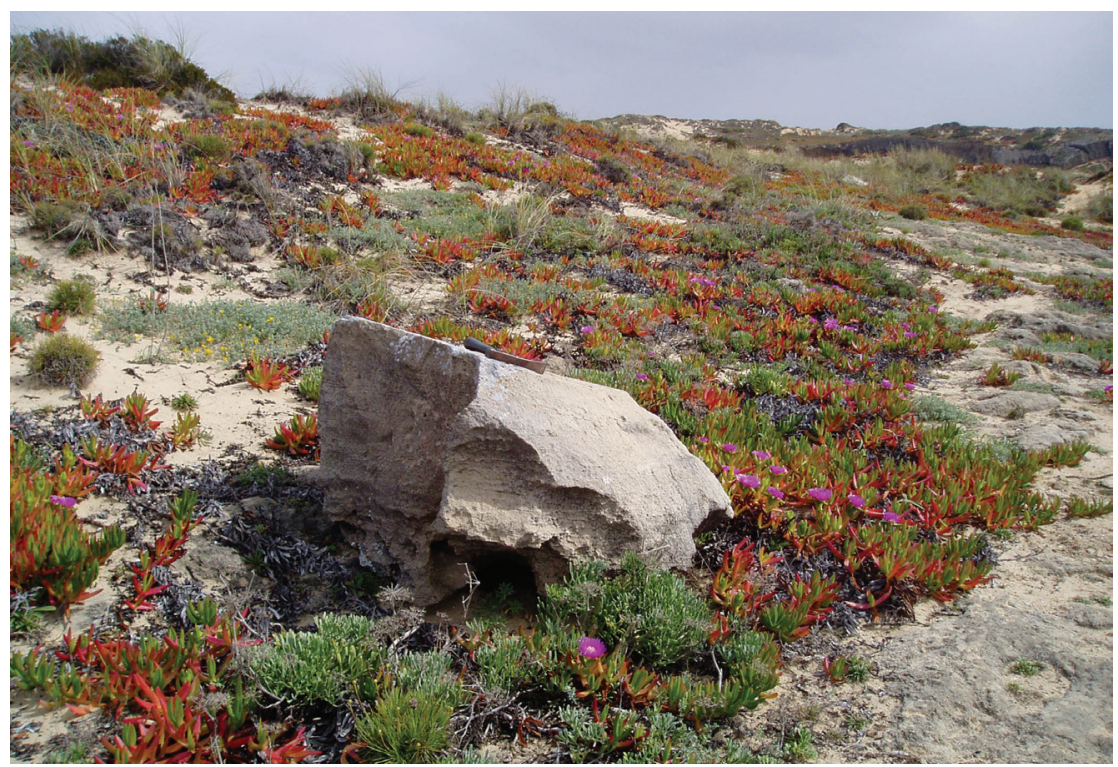

Fot. 5 - Um dos grandes blocos encontrados em posição invertida. O eolianito na parte inferior do bloco tem claras marcas de meteorização subaérea.

Photo. 5 - One of the boulders found in the inverted position. The eolianite boulder bottom surface has clear marks of subaerial weathering.

QUADRO I - Parâmetros dos blocos de maior dimensão em Malhão. R - rampa, $\mathrm{H}_{\mathrm{t}}$ - altura da onda de tsunami, $\mathrm{H}_{\mathrm{s}}$ - altura da onda de temporal.

TABLE I - Parameters of larger boulders. $R$-rampa, Ht-tsunami wave height, $H s$ storm wave height.

\begin{tabular}{|c|c|c|c|c|c|c|c|}
\hline Bloco & $\begin{array}{c}\text { Altitude } \\
(\mathbf{m})\end{array}$ & $\begin{array}{c}\text { Comando da } \\
\text { arriba }(\mathbf{m})\end{array}$ & $\begin{array}{c}\text { Distância à linha } \\
\text { de costa }(\mathbf{m})\end{array}$ & $\begin{array}{c}\text { Peso } \\
(\mathbf{t o n})\end{array}$ & $\begin{array}{c}\text { Volume } \\
\left(\mathbf{c m}^{\mathbf{3}}\right)\end{array}$ & $\begin{array}{c}\mathbf{H}_{\mathbf{t}} \\
(\mathbf{m})\end{array}$ & $\begin{array}{c}\mathbf{H}_{\mathbf{s}} \\
(\mathbf{m})\end{array}$ \\
\hline 4 & 12,12 & 12,12 & 0,00 & 13,61 & 7370000,00 & 5,27 & 21,07 \\
\hline 25 & 14,67 & $\mathrm{R}$ & 115,80 & 19,04 & 9490000,00 & 6,69 & 26,78 \\
\hline 27 & 16,49 & $\mathrm{R}$ & 80,80 & 18,23 & 9230000,00 & 6,02 & 24,09 \\
\hline
\end{tabular}


Note-se, que para os três casos (QuAdro I), a altura da onda de tsunami prevista pelo modelo de J. Nott (2003) se enquadra nas alturas determinadas para o litoral sul português, que, como já foi referido, se podem encontrar entre os 4,28m e os 10-12m. Blocos com o peso dos encontrados em Malhão, às altitudes e distâncias da linha de costa apontadas, necessitariam de ondas de temporal de altura improvável na costa portuguesa e o seu run-up já teria destruído grande parte do campo dunar de areia solta (saliente-se que este campo dunar de areia solta é também herdado, pois não existe hoje a área de alimentação em areias).

O temporal Hércules que assolou o litoral português em janeiro de 2014 ajuda a calibrar os dados obtidos. Durante esse temporal foi registada, na bóia ondógrafo de Sines, uma altura de onda máxima de $17 \mathrm{~m}$. Esta foi capaz de arrancar um bloco de eolianito ao substrato (ou face da arriba) e colocá-lo a $15 \mathrm{~m}$ de altitude, a cerca de $120 \mathrm{~m}$ da linha de costa. Embora não tenha sido avaliada a sua densidade, o eolianito deste bloco parece ser muito menos denso do que os restantes blocos (menos pesado) e de menores dimensôes (foto 6), encontrando-se fragmentado. Assim, as ondas do temporal Hércules apenas deslocaram um pequeno bloco, menos pesado e que náo penetrou tanto para o interior como os restantes (foto 6).

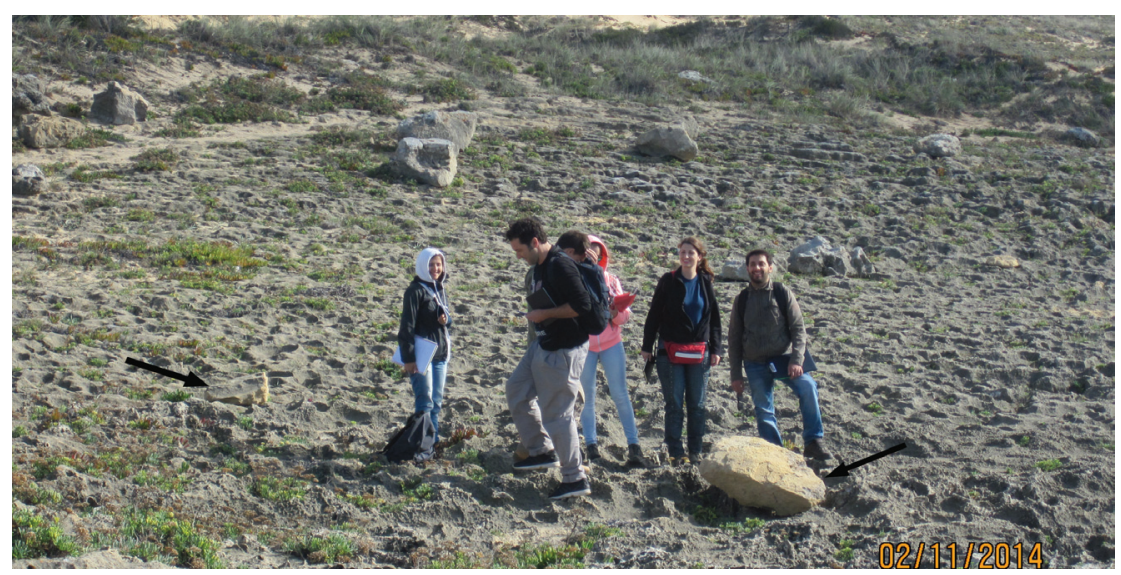

Fot. 6 - Bloco fragmentado de eolianito atirado pelo temporal Hércules para a rampa de Malhão. As setas indicam os dois fragmentos.

Photo. 6 - Fragmented boulder of eolianite thrown by the storm Hercules to the ramp of Malhão. The arrows indicate the two fragments. 
A micromorfologia do esporão de Malhão é também complexa. A superfície do eolianito está acidentada por pequenos sulcos e por depressóes circulares de pequenas dimensões de erosão mecânica. Tendo em consideração a morfologia sintetizada por E. Bryant (2008) e G. Goff (2009) para as superfícies atuadas por tsunamis, reconheceram-se em Malhão as seguintes formas (fot. 7 e fig. 4): arribas truncadas (A), rampas (B), grutas (C); promontório em flauta (D), mini canhōes de drenagem (E); sulcos transversais; entalhes sinuosos (F), numa superfície pontuada de buracos decimétricos de erosão mecânica.

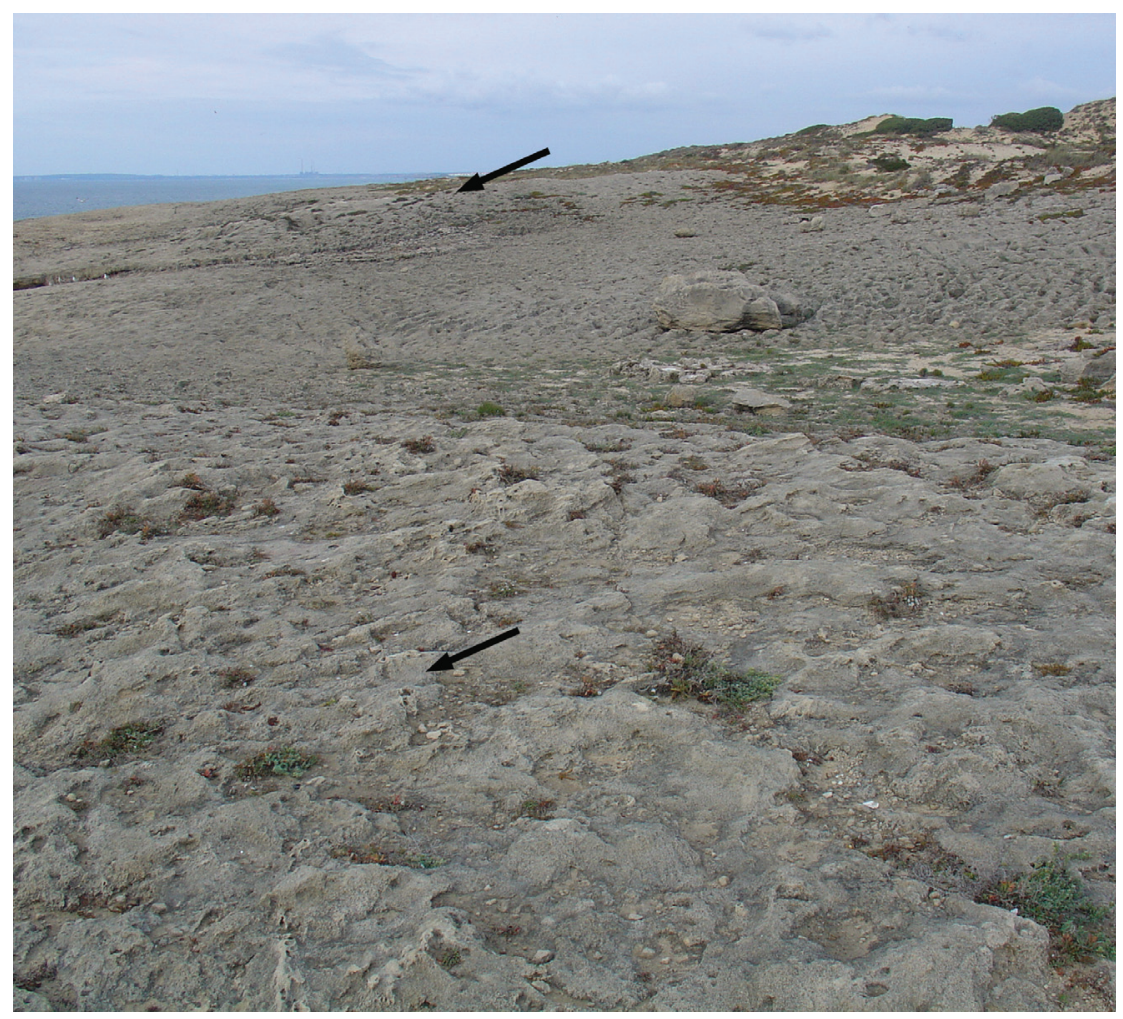

Fot. 7 - Micromorfologia da rampa de Malhão. As setas assinalam os promontórios em flauta, entre os quais a superfície do eolianito, subjacente aos grandes blocos, está sulcada transversalmente. Aproximadamente ao centro da imagem situa-se o bloco de 19 toneladas.

Photo. 7 - Malhão ramp micromorphology. The arrows indicate the headlands in flute, among which the surface of eolianite underlying the boulders is transversely grooved. The 19 tons boulder is at about the center of the image. 

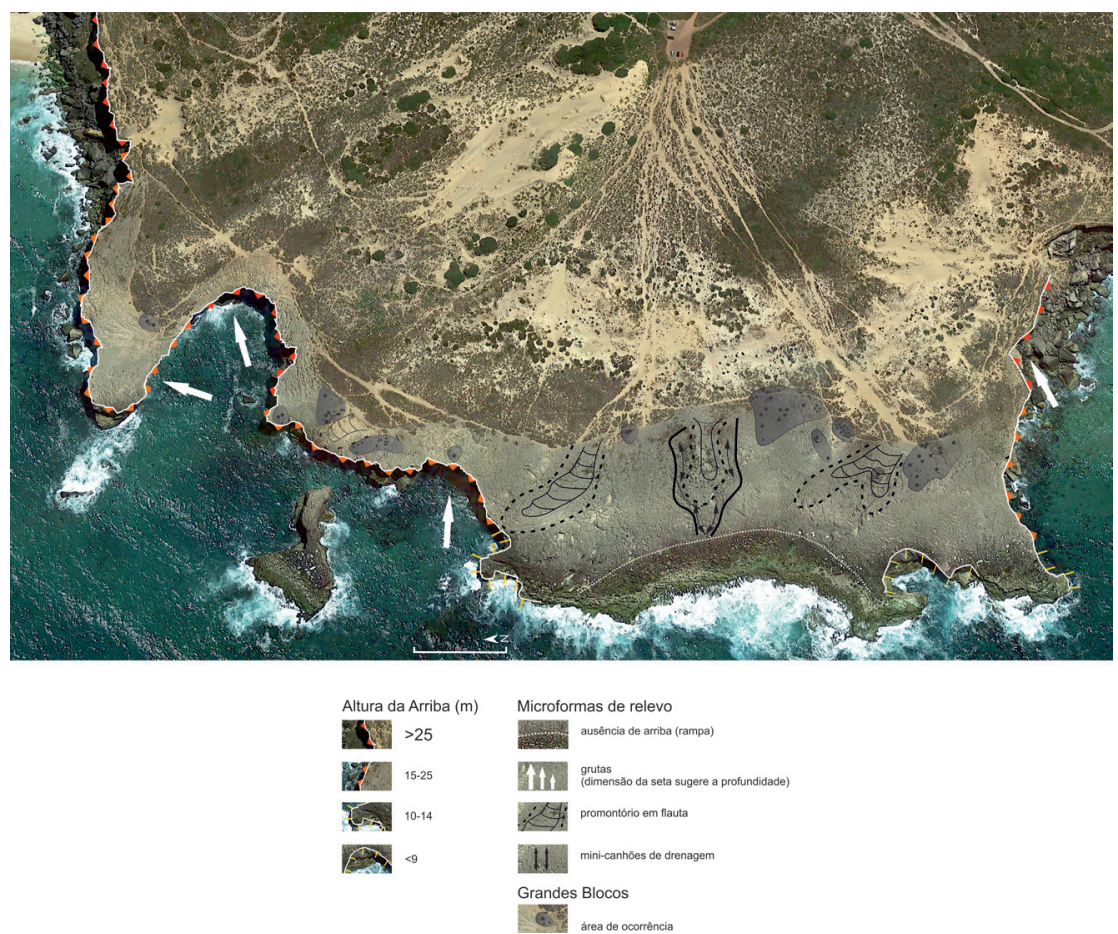

Fig. 4 - Geomorfologia do promontório de Malhão.

Fig. 4 - Malhão headland geomorphology.

\section{Conclusão}

Esta contribuição visa tâo somente chamar a atenção para um fenómeno extremo de efeito destruidor que ocorreu na costa portuguesa. Volvidos 260 anos sobre o terramoto e tsunami de 1755 , o litoral mudou muito de fisionomia. A nível nacional, este território concentra cerca de $75 \%$ da população, é responsável pela produção de $85 \%$ do produto interno bruto, nele se localizam as principais áreas urbanas e industriais, as áreas de turismo intensivo, que alternam com áreas naturais, rurais e de pesca. Estima-se que cerca de 30\% da costa portuguesa esteja ocupada com construçôes.

Algumas das áreas onde têm vindo a ser registados vestígios do tsunami de 1755 são atualmente marcadas por extensas e densas urbanizaçóes, resorts $e$ marinas, 
como sucede em Lisboa e em alguns locais do Algarve, com exceção das áreas costeiras protegidas, mas sobre as quais é grande a ambição de alguns agentes económicos, como já sucedeu por diversas vezes no litoral da Costa Vicentina.

Existem vários instrumentos de gestão territorial que condicionam o uso dos espaços litorais que não devem deixar de ser aplicados, por razóes economicistas, de "interesse nacional". O tipo de fenómeno tratado é de elevada magnitude, apesar da sua baixa frequência de ocorrência. Porém, não deve ser negligenciado porque, dado o contexto de Portugal continental, este fenómeno pode repetir-se, com consequências ainda mais devastadoras, com trabalho acrescido e difícil para os agentes da proteção civil.

\section{Referências bibliográficas}

Andrade, César (1990). Estudo da susceptibilidade ao galgamento da Ria Formosa. Geolis, vol. IV $(1,2)$, p. 69-76.

Andrade, César (1992). Tsunami generated forms in the Algarve barrier islands (South Portugal). Science of Tsunami Hazard, no 10, p. 21-33.

Andrade, César; Andrade, A. M., Kortekaas, Stella; Dawson, Alistair G. (1997). Sedimentological traces of tsunamigenic overwash of the Martinhal lowland. Seminário sobre a Zona Costeira do Algarve. Littoral '97. Proceedings, International Symposium Eurocoast, Faro, p. 11-17.

Araújo-Gomes, João (2013). Deformações em sedimentos finos não consolidados interpretados como sismitos. Finisterra - Revista Portuguesa de Geografia, Lisboa, vol. XLVIII, no 95, p.125-138.

Borges, José F.; Fitas, Augusto J.S.; Bezzeghoud, Mourad; Teves-Costa, Paula, (2001). Seismotectonics of Portugal and its adjacent Atlantic area. Tectonophysics, vol. 337, p. 373-387.

Baptista, Maria Ana; Miranda, Pedro M., Miranda, J. Miguel, Mendes-Victor, L. (1996). Rupture extent of the 1755 Lisbon earthquake inferred from numerical modelling of tsunami data. Phys. Chem. Earth, 21 (12), p. 65-70.

Baptista, Maria Ana; Heitor, S. C.; Miranda, J. Miguel; Miranda, Pedro; Mendes-Victor, Luiz (1998). The 1755 Lisbon Tsunami: evaluation of the tsunami parameters. Journal of Geodynamics. 25 (2), p.143-157.

Baptista, Maria Ana, Miranda, Pedro M.; Chierici, Francesco; Zitellini, Nevio (2003). New study of the 1755 earthquake source based on multi-channel seismic survey data and tsunami modelling. Natural Natural Hazard Earth Science, Systems 3, p. 333-340.

Bryant, Eduard A. (2008). Tsunami. The Underrated Hazard. Cambridge University Press, 2nd ed., Cambridge.

Costa, Pedro J. M.: Andrade, César; Cascalho, Joao; Dawson, Alistair, G.; Freitas, M. Conceição; Paris, Raphaël; Dawson, Stella (2015). Heavy mineral assemblages of onshore palaeotsunami sediments. The Holocene, 25(5), p. 795-809. 
Costa, Pedro; Andrade, César; Oliveira, Maria Alexandra; Taborda, Rui e da Silva, C. M. (2008). High energy Boulder deposition in Barranco and Furnas lowlands, western Algarve (south Portugal). Second International Field Symposium, IGCP Project 495, GI ${ }^{2} S$ Coast Research Publication, 6, p. 19-22.

Dawson, Alistair G.; Hindson, R. A.; Andrade, César; Freitas, Conceiçăo; Parish, Richard; Bateman, M. (1995). Tsunami sedimentation associated with the Lisbon earthquake of 1 November AD 1755: Boca do Rio, Algarve, Portugal. Holocene, 5 (2), p. 209-215.

Goto, Kazuhisa; Okada, Kiyohiro; Imamura, Fumuhiko (2009). Characteristics and hydrodynamics of boulders transported by storm waves at Kudaka Island, Japan. Marine Geology, 262, p.14-24.

Goff, J.; Dominey-Howes, D. (2014). Tsunami. In Treatise on Geomorphology. Reference Module in Earth Systems and Environmental Sciences, Volume 13: Geomorphology of Human Disturbances, Climate Change, and Natural Hazards, p. 204-218.

Hindson, R. A.; Andrade, César; Dawson, Alistair G. (1996). Sedimentary processes associated with the tsunami generated by the 1755 Lisbon earthquake on the Algarve coast, Portugal. Physics and Chemistry of the Earth, 21, p. 57-63.

Hindson, R. A.; Andrade, César (1999). Sedimentation and hydrodynamic processes associated with tsunami generated by 1755 Lisbon earthquake. Quaternary International, 56, p. 27-38.

Kortekaas, Stella; Dawson, Alistair G. (2007). Distinguish tsunami and storm deposits: an example from Martinhal, SW Portugal. Sedimentary Geology, 200, p. 208-221.

Ramos-Pereira, Ana (1987). Acumulaçóes arenosas eólicas consolidadas do litoral do Alentejo e Algarve ocidental. Centro de Estudos Geográficos, Linha de Acçáo de Geografia Física, L.A.G.F. no 27, Lisboa.

Ramos-Pereira, Ana e Angelucci, Diego E., (2004). Formaçôes dunares no litoral português, do final do Plistocénico e inícios do Holocénico, como indicadores paleoclimáticos e paleogeográficos. In: Tavares, A.A., Tavares, M. J. F. \& Cardoso, J. L. (Eds.), Evolução Geohistorica do Litoral Português e Fenómenos Correlativos, Universidade Aberta, Lisboa, p. 221-256.

Ramos-Pereira, Ana; Trindade, Jorge; Neves, Mário; Borges, Bruno (2009). Indicadores geomorfológicos de tsunami no Parque Natural do SW Alentejano e Costa Vicentina (Malhão). Publicaçôes da Associação Portuguesa de Geomorfólogos, vol VI, APGeom, Braga, p. 51-56.

Ramos-Pereira, Ana; Trindade Jorge; Araújo-Gomes, João (2013). Seismite and tsunamiite in urban environment (Lisbon). Finisterra - Revista Portuguesa de Geografia, Lisboa, vol. XLVIII, no 95, p. 141-147.

Ramos-Pereira, Ana; Araújo-Gomes, João e Trindade, Jorge (2014). Vestígios do tsunami de 1755, na zona de Belém ou o que esconde o novo Museu dos Coches. Rossio estudos de Lisboa, n. ${ }^{\circ}$, Issue online edition. Câmara Municipal de Lisboa.

Ribeiro, António; Cabral, João; Baptista, R.; Matias, Luís (1996) - Tectonic stress pattern in Portugal Mainland and the adjacent Atlantic Region. Tectonics, 15, p. 641-659.

Scheffers, Anja e Kelletat, Dieter (2005). Tsunami relics on the coastal landscape west of Lisbon, Portugal. Science of Tsunamis, vol. 23, no 1, p.3-16.

Shah-Hosseini, M.; Morhange, C.; De Marco, A.; Wante, J.; Anthony, E. J.; Sabatier, F.; Mastronuzzi, G.; Pignatelli, C.; Piscitelli, A. (2013). Coastal boulders in Martigues, French Mediterranean: evidence for extreme storm waves during the Little Ice Age. Zeitschrift für Geomorphologie, Supplementbände Volume 57 Supplementary Issue 4, p. 181-199

Scheffers, A.; Kelletat, D., (2004). Bimodal tsunami deposits - a neglected feature in paleotsunami research. Coastline Reports, 1, p. 67-75. 
Terrinha, Pedro; Pinheiro, Luís M.; Henriet, Jean-Pierre; Matias, Luís; Ivanov, M. K.; Monteiro, J. Hipólito; Akhmetzhanov, A.; Volkonskaya, A.; Cunha, T.; Shaskin, P.; Rovere, M. (2003). Tsunamigenic-seismogenic structures, neotectonics, sedimentary processes and slope instability on the southwest Portuguese Margin. Marine Geology 195, p. 55-73.

Trog, Carmen; Hempel, Rita; Frenzel, Peter; Mäusbacher, Roland (2015). Holocene palaeoenvironmental changes in three lagoons on the Algarve coast of Portugal. Palaeobio Palaeoenv, 95, p. 203-221.

Trog, Carmen; Höfer, Dana; Frenzel, Peter; Camacho, Sarita; Schneider, Heike; Mäusbacher, Roland (2013). A multi-proxy reconstruction and comparison of Holocene palaeoenvironmental changes in the Alvor and Alcantarilha estuaries (southern Portugal). Revue de Micropaléontologie, 56, p. $131-158$

Vött, A.;.Lang, F.; Brückner, H.; Gaki-Papanastassiou, K.; Maroukian, H.; Papanastassiou, D.; Giannikos, A.; Hadler, H.; Handl, M.; Ntageretzis, K.; Willershäuser, T.; Zander, A. (2011). Sedimentological and geoarchaeological evidence of multiple tsunamigenic imprint on the Bay of Palairos-Pogonia (Akarnania, NW Greece). Quaternary International, Volume 242, Issue 1, p. 213-239.

\section{Agradecimento}

Os autores desejam deixar expresso o seu agradecimento aos revisores científicos deste capítulo, cujas sugestôes em muito contribuíram para o melhorar. 